\title{
The Role of Deep Learning Method Based on Environmental Geochemical Data in Resource
}

\author{
Pei $\mathrm{Yao}^{1, *}$ \\ ${ }^{1}$ College of Geo-exploration Science and Technology, Jilin University, Changchun, China
}

\begin{abstract}
In recent years, with the continuous development of artificial intelligence, deep learning, as an important method of artificial intelligence learning, has made great progress. At present, deep learning has been successfully applied in many engineering fields. Environmental science itself involves a wide range, among which environmental geochemistry is an important branch. The combination of environmental geochemical problems and deep learning can better study the role of geochemical problems in environmental investigation, and also can use the basic situation of regional environmental elements to predict mineral resources.
\end{abstract}

\section{Introduction}

The so-called environment is the synthesis of various factors for human survival and development [1]. The application of Geochemistry in the field of environment can involve many aspects, mainly including the following two aspects: one is the original environment directly related to the geological background; the other is the environment participated by the products of human activities. An important purpose of environmental geochemical survey is to determine the environmental background and its changing trend, and monitor it regularly, so as to provide scientific data for the protection of human living environment and sustainable development. Soil is the main medium of environmental geochemical element survey. Chemical pollutants in soil and sediment enter food and cause human food chain poisoning. However, it is less affected by human activities, and the regional environmental indicators exceed the standard, which is mostly caused by the natural geological evolution. Some elements in the soil are enriched to a certain extent, which may be related to the occurrence of the deposit.

With the rapid development of deep learning, the application field has expanded from the traditional natural speech processing, image recognition and speech recognition to big data analysis and other fields [2]. At present, a variety of deep learning tools have been developed, and a variety of optimization methods and training methods for deep learning have been proposed in the field of machine learning. GoogLeNet is a deep learning structure proposed by Christian Szegedy in 2014. Figure 1 shows the basic structure of the induction module. Multiple such induction modules are connected in series to form the whole induction structure [3]. There are two main contributions of the initiation structure: one is to perform convolution re aggregation on multiple dimensions at the same time; the other is to use convolution for dimension lifting. The theory and practicability of this method have been explained in detail in the original text, and this paper will not be repeated due to the limitation of space.

Deep learning is not a sudden technology, but is realized by multiple small technological advances [4] [5]. In this paper, the method of deep learning is used to learn the soil geochemical elements corresponding to the known deposit location in the region, and then other unknown areas are learned.

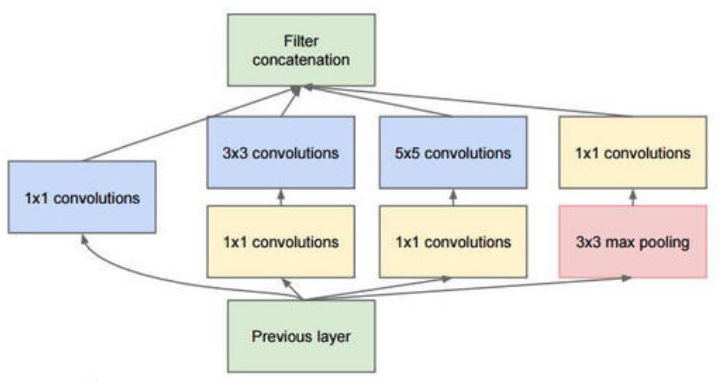

Fig. 1. basic structure of the inception module

\section{EXPERIMENTAL DESIGN}

\subsection{Sample data}

The data used in this study is 1:50000 soil geochemical data of a certain area in Gansu Province. As a polymetallic metallogenic belt, the $\mathrm{Pb}$ element in the study area can not only indicate the impact of heavy metal elements on the environment, but also indicate the location of lead-zinc deposits in this area. Therefore, we use these data to predict the mineral resources in the unknown area. First, we use triangulation method to grid

\footnotetext{
$\overline{\text { * Corresponding author: peiyao@jlu.edu.cn }}$
} 
the data to form the contour map of $\mathrm{Pb}$ element distribution, and divide it into 551 sample images. In these images, there are 288 images with lead-zinc deposits, 263 images without lead-zinc deposits, and the yellow dots in the images with lead-zinc deposits are the location of the deposits.

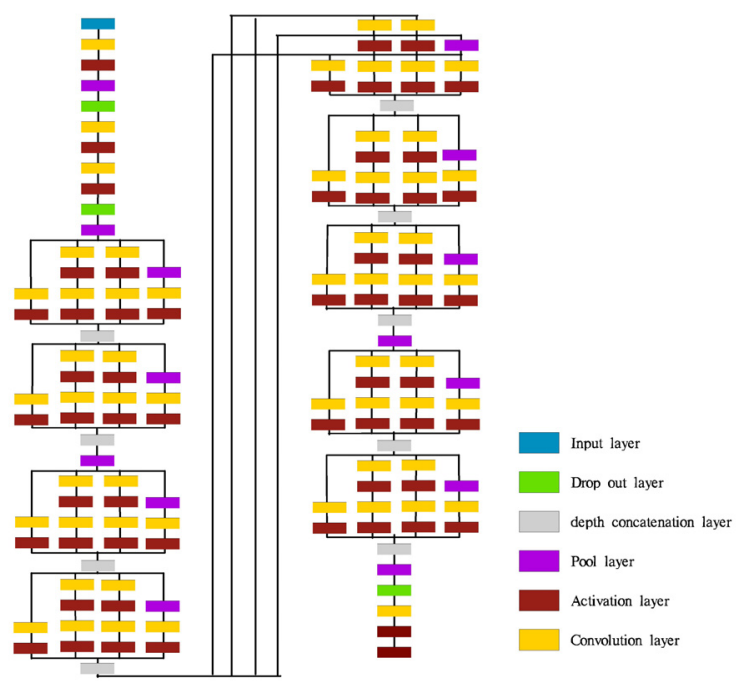

Fig. 2. Structure diagram of GoogLeNet model

\subsection{Data enhancement}

The learning of artificial neural network depends on a large number of training data, the quality of training set and the number of training samples will have a great impact on the performance of neural network [6]. Using mirror flip is to flip the original image up and down and left and right. In the corresponding pixel data matrix, if the image is flipped up and down, it shows that the ordinates of all the pixels are symmetrical with the ordinates of the corresponding pixels in the original image about the horizontal centerline of the image. In the same way, left and right flipping means that the abscissa of all the pixels after the left and right mirror flipping operation is symmetrical with the abscissa of the corresponding pixel in the original image about the vertical centerline of the image.

Using image enhancement, the original image data is enhanced, which greatly expands the number of input images in the training set.

\section{3 model training and analysis}

The training model used in this study has the following main parameters: the learning rate is 0.0001 , the maximum epoch is 3 , and the selection of validation frequency is 5 . After 8 rounds of training with MATLAB software, the training accuracy is $80 \%$, the verification accuracy is $79 \%$, and the cross entropy is 0.05 . The training process and main parameter results can be seen from the curve in Figure 4. The whole training process adopts single GPU, and the training time lasts for 2 minutes and 56 seconds. It can be seen from the above parameters that the deep learning framework and training precision adopted in this work are high, which can play a role in the prediction of mineral resources by using soil geochemical data in this study area.

\section{Test group image prediction}

Randomly selected 200 images are taken as the test group, including 100 images with ore and 100 images without ore. Some sample images are shown in Figure 5. The selected test image is input into neural network for recognition, and some results are shown in Table 1.

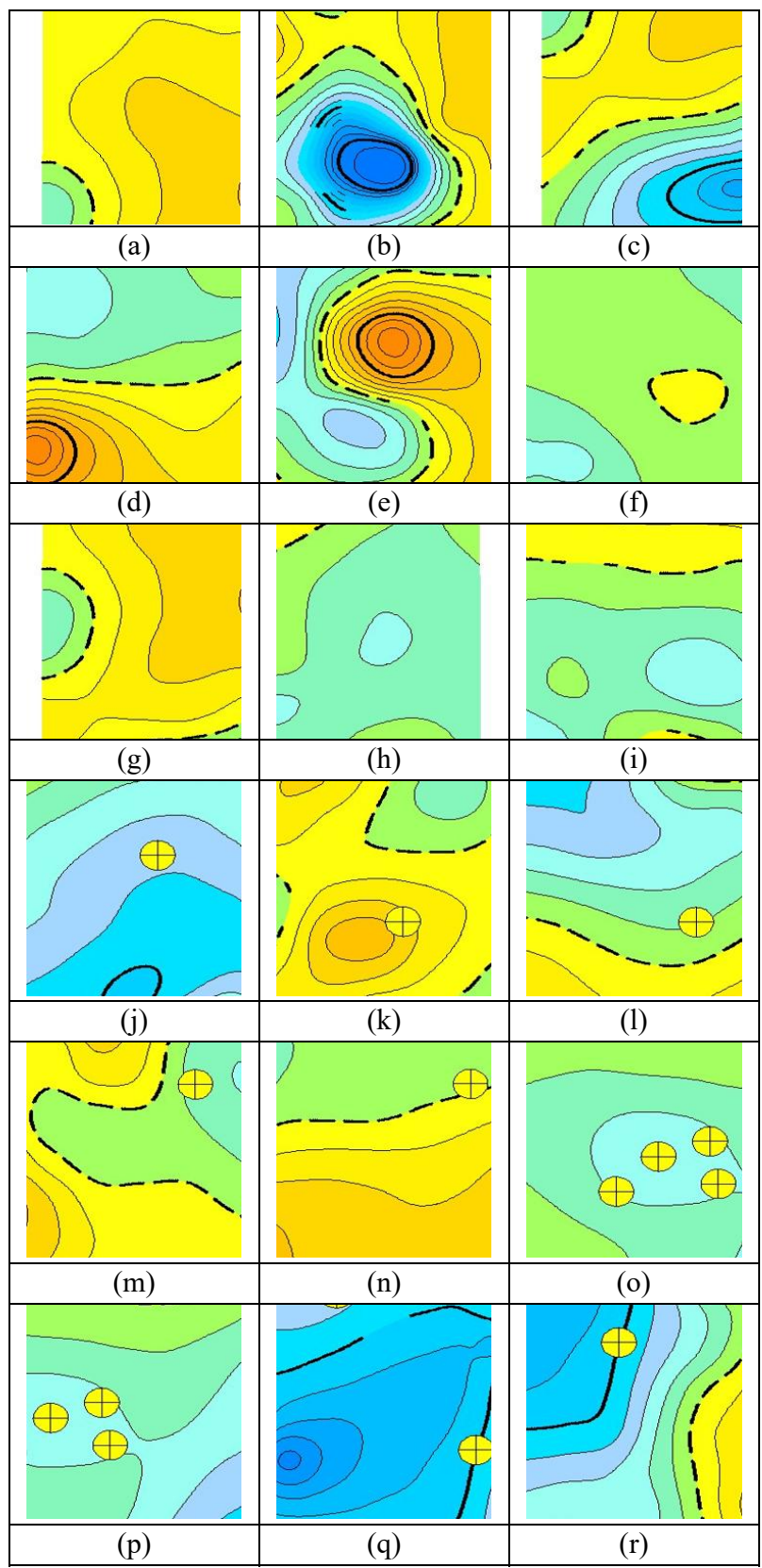

Fig. 3. Structure diagram of GoogLeNet model (a) - (i) non ore image; (j) - (r) ore image 


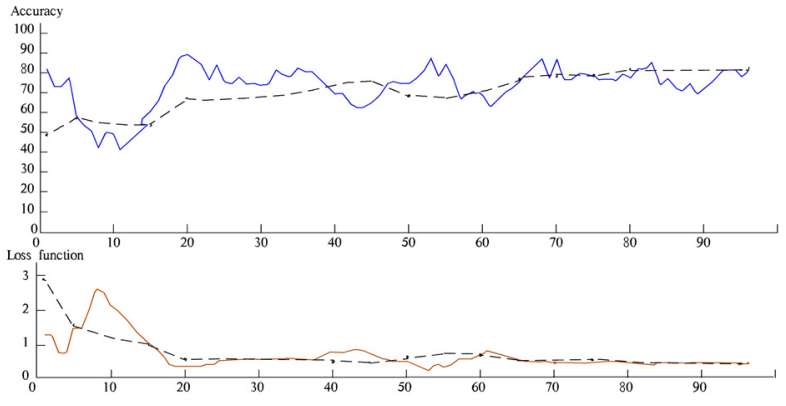

Fig. 4. Image recognition training process (blue - training accuracy; Brown - cross entropy; black - test accuracy)

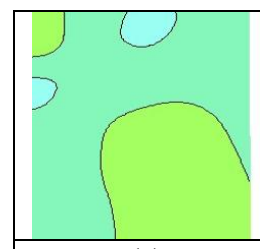

(a)

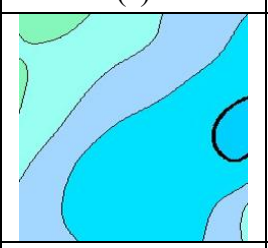

(d)

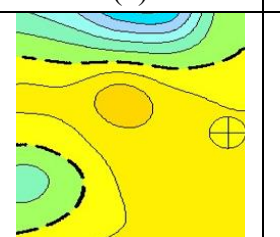

(g)

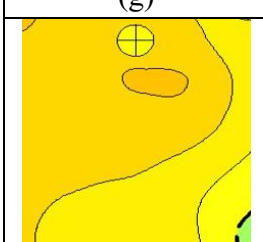

(j)

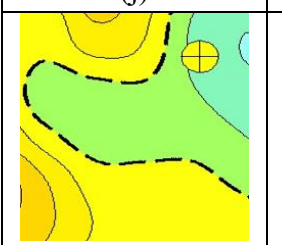

(m)

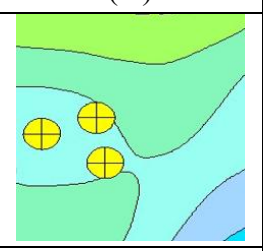

(p)

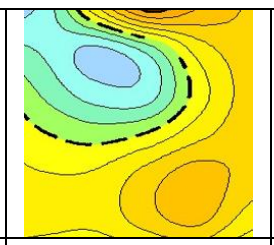

(b)

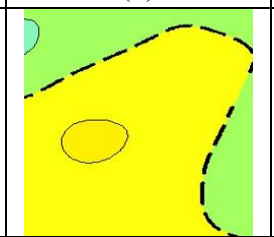

(e)

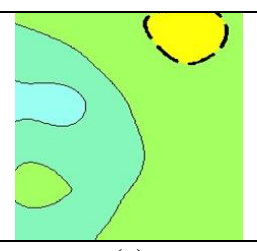

(c)

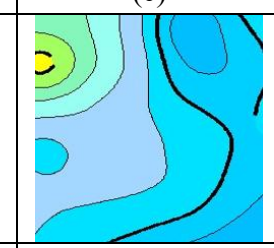

(f)

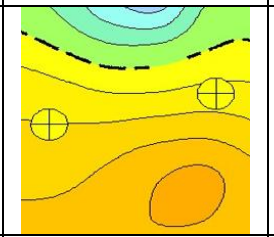

(h)

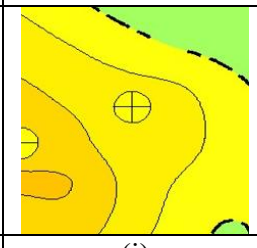

(i)

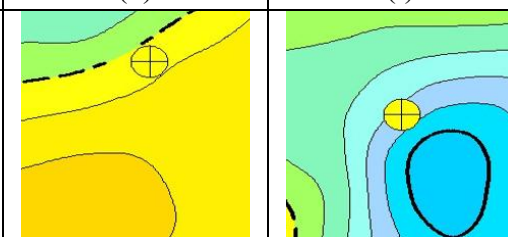

(1)

\section{(k)}

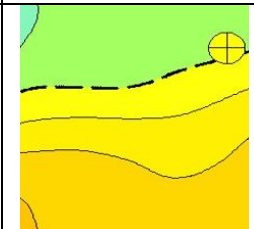

(n)

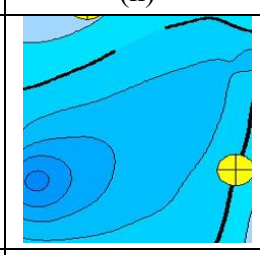

(q)

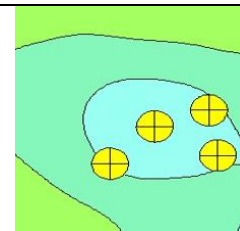

(o)

(r)

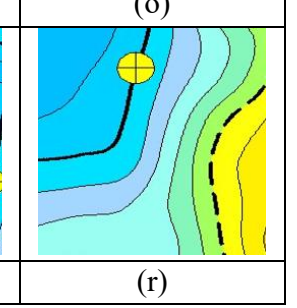

. 5xample of some image test samples

(a) - (i) non ore image; (j) - (r) ore image

As shown in Table 1, there are 310 images in the test set. The correct results are obtained through recognition, and the recognition accuracy is $77 \%$. The image of the test set is divided into two parts: one is the ore bearing image, the recognition rate is $78 \%$; the other is the non ore bearing image, the recognition rate is $76 \%$. A total of 270 correct images and 40 fault images were identified.

Table 1. Results of metallogenic prediction and recognition of some images

\begin{tabular}{|c|c|c|c|c|}
\hline \multirow[t]{2}{*}{$\begin{array}{c}\text { Test } \\
\text { image }\end{array}$} & \multicolumn{2}{|c|}{$\begin{array}{c}\text { Classification } \\
\text { recognition } \\
\text { probability } \\
(\%)\end{array}$} & \multirow[t]{2}{*}{$\begin{array}{c}\text { Is it } \\
\text { mineralized } \\
?\end{array}$} & \multirow[t]{2}{*}{$\begin{array}{c}\text { Accurac } \\
\mathrm{y}\end{array}$} \\
\hline & Ore & $\begin{array}{l}\text { Non } \\
\text { ore }\end{array}$ & & \\
\hline Test-1 & 0.98 & 0.02 & no & ture \\
\hline Test-2 & 0.93 & 0.07 & no & ture \\
\hline Test-3 & 0.66 & 0.34 & no & ture \\
\hline Test-4 & 0.71 & 0.29 & no & ture \\
\hline Test-5 & 0.58 & 0.42 & no & ture \\
\hline Test-6 & 0.14 & 0.86 & no & fault \\
\hline Test-7 & 0.76 & 0.24 & no & ture \\
\hline Test- 8 & 0.89 & 0.11 & no & ture \\
\hline Test-9 & 0.26 & 0.74 & no & fault \\
\hline Test-10 & 0.55 & 0.45 & no & ture \\
\hline Test-11 & 0.79 & 0.21 & no & ture \\
\hline Test-12 & 0.69 & 0.31 & no & ture \\
\hline Test-13 & 0.88 & 0.12 & no & ture \\
\hline Test-14 & 0.74 & 0.26 & no & ture \\
\hline Test-15 & 0.86 & 0.14 & no & ture \\
\hline Test-16 & 0.63 & 0.37 & no & ture \\
\hline Test-17 & 0.45 & 0.55 & no & fault \\
\hline Test-18 & 0.34 & 0.66 & no & fault \\
\hline Test-19 & 0.47 & 0.53 & no & fault \\
\hline Test-20 & 0.53 & 0.47 & no & ture \\
\hline Test-21 & 0.88 & 0.12 & no & ture \\
\hline Test-22 & 0.78 & 0.22 & no & ture \\
\hline Test-23 & 0.89 & 0.11 & no & ture \\
\hline Test-24 & 0.77 & 0.23 & no & ture \\
\hline Test-25 & 0.59 & 0.41 & no & ture \\
\hline Test-26 & 0.30 & 0.70 & no & fault \\
\hline Test-27 & 0.51 & 0.49 & no & ture \\
\hline Test-28 & 0.76 & 0.24 & no & ture \\
\hline Test-29 & 0.87 & 0.13 & no & ture \\
\hline Test-30 & 0.64 & 0.36 & no & ture \\
\hline
\end{tabular}


Deep learning requires a high amount of data, because the amount of data plays a very important role in the learning effect of the model. When a certain type of image data is less, such as the data of non ore image in the training set is less, it will have a great impact on the prediction effect of non ore image in the test set, leading to the phenomenon of prediction error. The number of training rounds will also affect the overall training effect. For example, this work only trained 8 rounds, reaching nearly $80 \%$ of the training accuracy. If the training rounds are increased, the training accuracy will also be greatly increased, but this will also lead to a great increase in the time of deep learning. For example, the training rounds will increase 5 times, and the same time will also increase 5 times. Therefore, high performance GPU, or GPU parallel computing, is an important tool and mainstream method for deep learning. In the followup work, in order to make the work of this paper more applicable, we should increase the use of highperformance GPU.

\section{Conclusion}

Based on deep learning method, this paper mainly studies the prediction of mineral resources using environmental geochemical data. The results show that the primary environmental geochemical pollution is mostly caused by geological evolution, and it is relatively possible to find mineral resources near the soil geochemical anomaly area. Through deep learning neural network training, the spatial relationship between geochemical anomalies and ore deposits is found.

Artificial neural network based on deep learning is a new research method in recent years, which has been successfully applied in many fields, especially in the field of image recognition. The level of deep learning for face recognition and object recognition has been very close to human. Other difficult image recognition has also made important progress, such as medical collision recognition.

However, the application of deep learning in environmental geochemistry has just started, and there are still many uncertain factors, so we need to continue to pay more attention to it. I believe that in the near future, deep learning in this field, theoretical research and application will be greatly improved.

\section{Acknowledgments}

This work was financially supported by Pilot demonstration of intelligent mineral geological survey in typical mining areas fund.

\section{References}

1. Jiang, J.Y. Cheng, J.P. Qi, S.H. Xiang, W. (2006) Applied Geochemistry. China University of Geosciences Press, Wu Han.

2. Yamashita T. (2018) Gpaphic Deep Learning. Posts Telecom Press, Bei Jing.
3. Szegedy C, Liu W, Jia Y., (2014) Going deeper with convolutions. 2015 IEEE Conference on Computer Vision and Pattern Recognition, 2014:1-9.

4. Hinton, G. E. Deng, L. Yu, D. Dahl, G. E. Mohamed, A. Jaitly, N. Senior, A. Vanhoucke, V. (2012) Deep neural networks for acoustic modeling in speech recognition. IEEE Signal Processing Magazine, 29(6), 82-97.

5. Simard, P. Y. Steinkraus, D. Platt, J. C. (2013) Best practices for convolutional neural networks applied to visual document analysis. In International Conference on Document Analysis and Recognition (ICDAR), 958-962.

6. $\mathrm{Xu} \mathrm{S.T,} \mathrm{Zhou} \mathrm{Y.Z,} \mathrm{(2018)} \mathrm{Artificial} \mathrm{intelligence}$ identification of ore minerals under microscope based on deep learning algorithm. Acta Petrologica Sinica, 34(11): 3244-3252. 